\title{
EXTRACELLULAR SMALL RNAS AND THEIR TRANSFER BETWEEN SPECIES AND KINGDOMS
}

Keywords: dsRNA, miRNA, siRNA, xenomiR, systemic RNAi, environmental RNAi

\section{PETR SVOBODA}

Institute of Molecular Genetics, Academy of Sciences of the Czech Republic, Videnska 1083, 14220 Prague 4, Czech Republic

Correspondence to: Petr Svoboda, Institute of Molecular Genetics ASCR, Videnska 1083, 14220 Prague 4, Czech Republic, tel. \# +420 241063147, e-mail: svobodap@img.cas.cz.

\begin{abstract}
RNA silencing is a common term for mechanisms where small RNAs guide repression of other RNAs (mRNAs, retrotransposon RNAs, viral RNAs) in a sequence-specific manner. RNA silencing exists among eukaryotic organisms where the core mechanisms evolved into many variants with different biological functions. The two most common RNA silencing pathways are microRNA (miRNA) and RNA interference (RNAi). While the miRNA pathway serves for negative regulation of endogenous genes, RNAi pathway usually functions as a form of protection against parasitic sequences although it also targets endogenous genes in some cases. Notably, RNA silencing does not often operate in a cell-autonomous manner. In a number of cases, small RNAs were detected in extracellular environment and were observed to pass through cells and even to be transferred between organisms. This review provides an overview of "mobile" small RNAs, with a particular focus on miRNAs and short interfering RNAs (siRNA) of the RNAi pathways, which exert their effects in different species than they originate from.
\end{abstract}

\section{Introduction}

RNA silencing utilizes small RNAs as sequence-specific guides for repression of cognate RNAs. The two most common small RNA pathways are microRNA (miRNA) and RNA interference (RNAi) pathways (Fig. 1). miRNA and RNAi are typically presented in a cell autonomous manner, i.e. repression remains restricted to cells directly exposed to dsRNA. However, small RNA-mediated repression can propagate across cell boundaries. There are two distinct modes of non-cell autonomous RNAi (Fig. 2, (Whangbo and Hunter, 2008)): (1) systemic RNAi includes processes where a silencing signal spreads from a cell across cellular boundaries into other cells and (2) environmental RNAi, which involves processes where dsRNA is taken up by a cell from the environment. Both modes can be combined in different ways. For example, gene silencing by soaking C. elegans in dsRNA solution is a combination 


\section{MiRNA}

gene repression

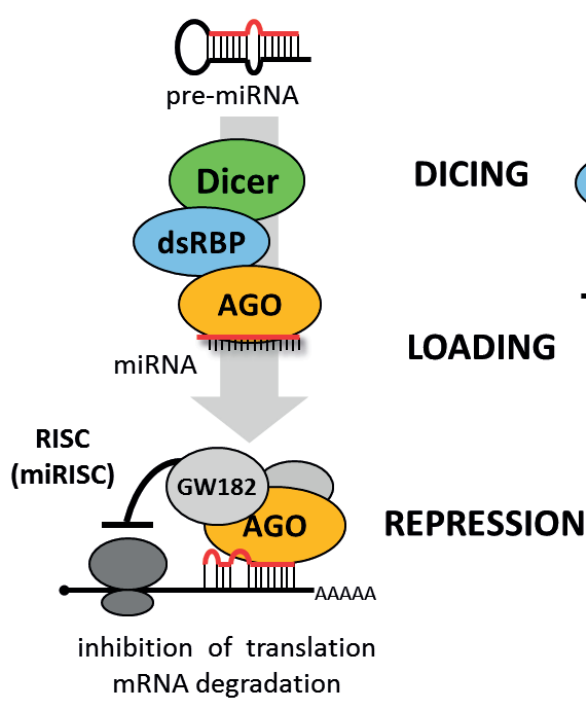

RNAi

viruses, convergent transcription, inverted repeat transcription, $\mathrm{RdRp}$ activity, artificial

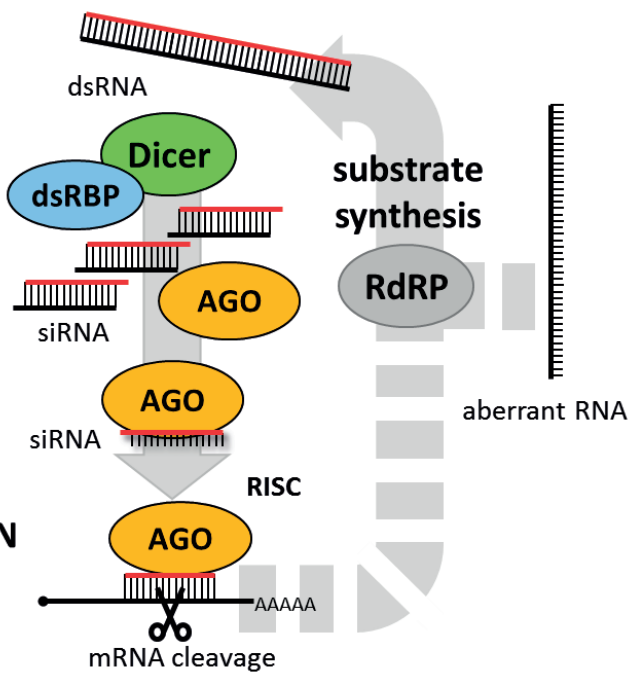

Figure 1 Schematic overview of miRNA and RNAi pathways. miRNA pathway is shown from the moment of miRNA precursor (pre-miRNA) processing by Dicer. miRNAs are genome-encoded and pre-miRNAs are produced from long primary transcripts with local small hairpin structures, which are processed to give rise to pre-miRNA. While both pathways show distinct modes of repression, miRNAs cully complementary to cognate mRNAs, which are common in plants but rare in mammals, can also induce RNAi-like cleavage. At the same time, siRNAs with partial complementarity can induce miRNA-like translational repression in mammalian cells. RdRP component of RNAi is present in some taxons (plants, nematodes) but some species execute RNAi without it (vertebrates, insects).

of environmental and systemic RNAi (Tabara et al., 1998). Non-cell autonomous RNAi has been discovered also in parasitic nematodes (Geldhof et al., 2007), hydra (Chera et al., 2006), planaria (Newmark et al., 2003; Orii et al., 2003), insects (Tomoyasu et al., 2008; Xu and Han, 2008), or plants (Himber et al., 2003). An extreme case would be trans-species (or even trans-kingdom) effect where dsRNA or small RNAs (miRNA or siRNA) produced in one organism (e.g. plant) would induce silencing in another organism (e.g. insect pest).

\section{Horizontal transfer of small RNAs and its possible consequences}

\section{Plant-pathogen transfer \& artificial plant resistance to pathogens}

RNA silencing can spread out of a plant as demonstrated by RNAi-inducing transgenes, which can extend their effects into nematodes (Huang et al., 2006), insects (Baum et al., 


\section{cell autonomous \\ RNA silencing}
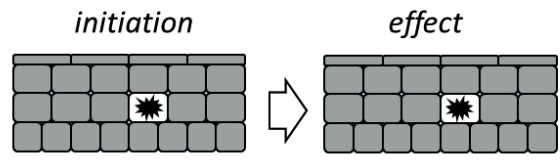

example

mammals
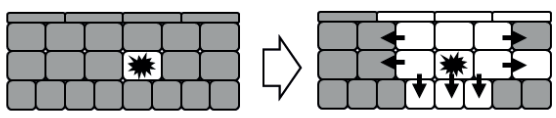

\section{C. elegans}

some Arthropods (Tribolium)

plants

RNA silencing

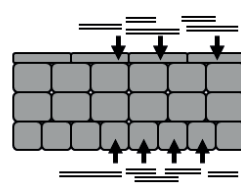

plant tissue

environmental

RNA silencing

trans-species

RNA silencing
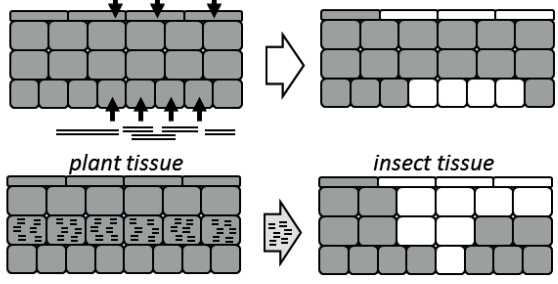

\section{C. elegans}

insects

\section{"RNAi-pesticide"}

Figure 2 Cell autonomous and cell non-autonomous RNAi

RNAi can either act in a cell autonomous manner, i.e. affecting only cells where RNA silencing was initiated. Cell non-autonomous RNA silencing types include systemic, where the RNAi effects propagates across cellular boundaries (e.g. mediated by transport of small RNAs) or environmental where the silencing RNA (e.g. dsRNA substrate) can be absorbed from the environment. Systemic and environmental RNAi can coexist and contribute to trans-species silencing where, for example, small RNAs produced in one species induce silencing in another one.

2007; Mao et al., 2007), parasitic fungi (Nowara et al., 2010) or another plant through a parasitic plant intermediate (Melnyk et al., 2011). Thus, trans-kingdom RNAi combining systemic \& environmental RNAi has biotechnological implications as it allows for producing a genetically engineered plant, which can be resistant to a specific virus or even induce an RNAi effect in a selected recipient (Mansoor et al., 2006). This can be explored, for example, for producing plants expressing dsRNA and selectively targeting RNAi-sensitive pests with an outcome of choice, e.g. repelling the pest, immobilizing it, sterilizing it (Bhatia et al., 2012), or killing it (Baum et al., 2007; Kola et al., 2016). Given the genome sequence diversity and relatively high sequence-specificity of RNAi, RNAi offers an adjustable selectivity for pest control. At the same time, this new technology might raise safety concerns. If the small RNAs can spread, could an RNAi-inducing transgene in a plant affect also non-targeted organisms?

The issue is essentially equivalent to off-targeting effects of RNAi brought to a species level. RNAi-off-targeting is a manifestation of the fact that partial base pairing can also induce RNA silencing. Consequently, some level of off-targeting in RNA silencing is inevitable. However, off-target effects can be suppressed to the point that they are not phenotypically manifested. The key factors reducing RNAi off-targeting effects in trans-kingdom RNAi are (i) the composition of the small RNA population (Fig. 3) and (ii) amount 
of small RNAs loaded on effector complexes, which would exhibit miRNA-like reduced sequence-specificity (i.e. seed match). It can be inferred directly from the molecular mechanism of RNAi and miRNA pathways that a homogeneous small RNA population where all small RNAs have the same sequence (e.g. represented by a single specific siRNA delivery) has a higher off-target risk than a mixture of small RNAs with different sequences (e.g. produced from a long dsRNA).

In transmission of RNA silencing from one species to another, the amount of small RNAs loaded on effector complexes in the recipient species will be influenced by (i) the nature of the transferred silencing molecule (small RNA, long dsRNA), (ii) efficiency of the transfer, and (iii) efficiency of effector complex formation in the recipient species. Thus, species, which can absorb long dsRNA and have systemic RNAi like C. elegans (Tabara et al., 1998) are more prone to exhibit specific silencing effects as well as off-target effects. At the same time, significant off-target effects require specific conditions in terms of stoichiometry between small RNAs and their targets, which are unlikely to be met upon RNAi induction with long dsRNA.

\section{Extracellular miRNAs in mammals and their transfer}

Release of miRNAs from mammalian cells is a well-known phenomenon. Here, I will highlight features of circulating miRNAs, which are important in the context of this review.

Extracellular circulating mammalian miRNAs were reported in 2008 when they were found in serum of lymphoma patients; they were immediately recognized as potential non-invasive biomarkers for cancer diagnostics and treatment (Lawrie et al., 2008). In fact, the bulk of the circulating RNA literature concerns biomarker potential of circulating miRNAs, which is of high clinical relevance but outside the scope of this report. Extracellular miRNAs were identified in a broad range of biological fluids (Baglio et al., 2015; Dismuke et al., 2015; Huang et al., 2013; Izumi et al., 2015; Kropp et al., 2014; Pegtel et al., 2011; Wu et al., 2015). Apart from plasma, circulating miRNAs in milk are also intensely studied (Alsaweed et al., 2016a, b, c; Benmoussa et al., 2016; Izumi et al., 2012; Izumi et al., 2015; Kuruppath et al., 2013; Sun et al., 2013; Xi et al., 2016).

miRNAs were identified in the cargo of exosomes, membranous vesicles 40 to $100 \mathrm{~nm}$ in diameter, which are constitutively released by almost all cell types and are found essentially in every biological fluid (reviewed, for example, in Rak, 2013; Yoon et al., 2014). Exosomal vesicles can carry miRNAs as well as siRNAs (reviewed in Lasser, 2012) - in the latter case, exosomes were adapted as a delivery tool for siRNAs (reviewed in Kumar et al., 2015). However, most individual exosomes in standard preparations do not seem to contain biologically significant numbers of miRNAs (Chevillet et al., 2014) and 95-99\% of circulating miRNA were reported to be associated with AGO proteins and not in extracellular vesicles (Arroyo et al., 2011; Turchinovich et al., 2011).

The molecular mechanism of miRNA release, whether as a cargo in a vesicle or not, is unclear and so is the function of circulating miRNAs, if there is any. Two distinct hypotheses were formulated concerning existence of circulating miRNAs (for a detailed review, see (Turchinovich et al., 2016)): 


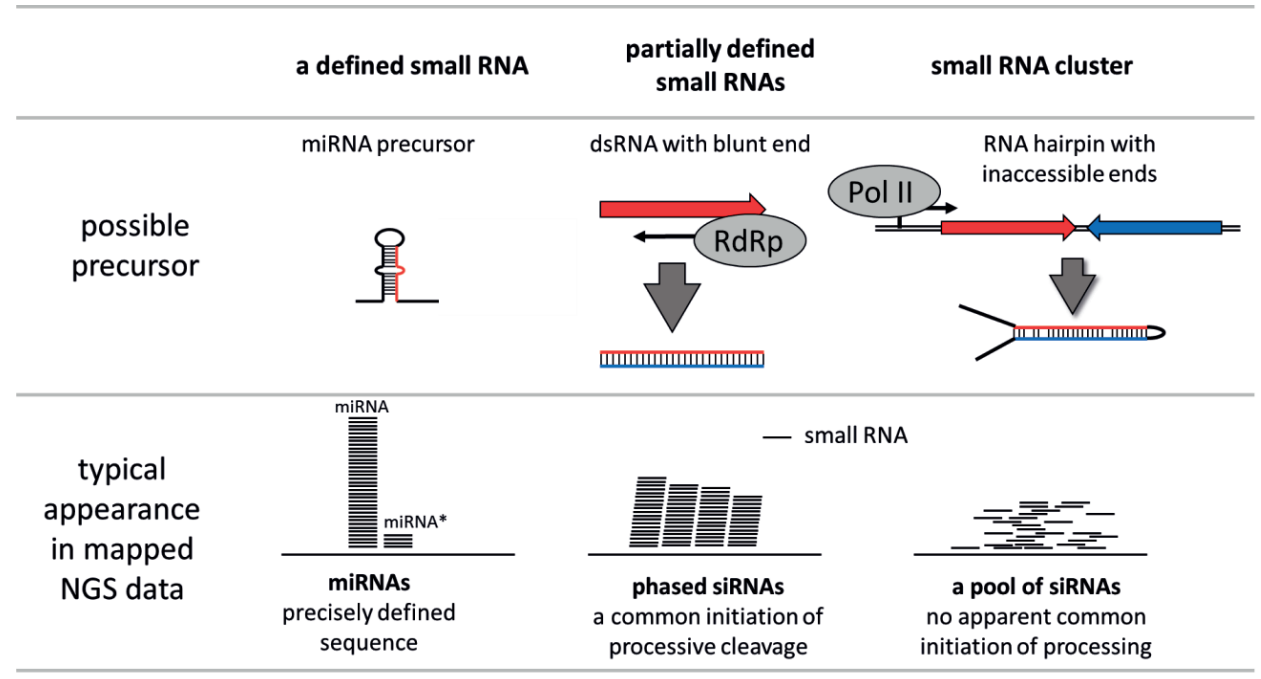

Figure 3 Different structures of small RNA populations from different precursors.

Two distinct types of small RNA populations exist: Highly homogeneous small RNAs, exemplified by miRNAs, which are present in cells in many (e.g. 103-104) identical copies, i.e. having the same sequence, which is "annotateable". Their biological effect on gene expression is partially predictable even if there is only a partial sequence complementarity (typically 6-7 nucleotides at the 5' end). The second type, exemplified by siRNAs derived from various dsRNA, is characterized by existence of populations of small RNAs with many different sequences, which originate from a longer dsRNA. Although such a population can be sequenced to identify individual sequences, their individual annotation is pointless and target prediction is restricted to high complementarity of the substrate dsRNA sequence.

Cell-to-cell communication hypothesis: According to this hypothesis, miRNAs can mediate cell-to-cell signalling. While the hypothesis is supported by circumstantial and correlative evidence (reviewed in Turchinovich et al., 2016), a mechanism for sorting, mobility, and targeting by mobile miRNAs and their function in vivo are unclear. This hypothesis has to face existing kinetic data (Wee et al., 2012) either by providing reliable evidence that sufficient quantities of miRNAs reach recipient cells or by identifying some non-canonical signaling function.

Cellular by-product hypothesis: This hypothesis states that circulating miRNAs are a non-specific noise resulting from cell physiology and turnover of biological material in the organism. As such, circulating miRNAs do not have a specific cell-to-cell communication function and their role in regulating gene expression in other cells is negligible or even non-existent. While this hypothesis might be consistent with the kinetic data (Wee et al., 2012), one cannot exclude a possibility that the "noise" of circulating miRNAs could yield specific biological roles during evolution. In fact, emergence of such an adaptation could be expected.

The scenario where the bulk of circulating miRNAs would represent a consequence of cell physiology while a limited number of circulating miRNAs would represent functionally relevant adaptations might reflect the real situation. In any case, extensive and 
rigorous research is needed to address the problem. This means in particular designing experiments aimed at proving either hypothesis wrong by deciphering underlying molecular mechanisms.

Finally, extracellular mammalian miRNAs can be transmitted from one animal to another or even between species. For example, placental miRNAs were found circulating in maternal plasma (Chim et al., 2008). It has also been reported that food-borne plant miRNAs are able to regulate gene expression in the liver (Zhang et al., 2012a) or miRNAs from consumed milk might contribute to circulating miRNAs in plasma (Baier et al., 2014) or that exosomes secreted by nematode parasites transfer small RNAs to mammalian cells (Buck et al., 2014). The issue of xenogenic miRNAs is addressed in detail next.

\section{Exogenic (nutritional, xenogenic) miRNAs in mammals}

In 2012, the article by Zhang et al. proposed that miRNAs from ingested plants could traverse into the bloodstream and suppress genes in the liver (Zhang et al., 2012a). The report sparked an ongoing debate because of potential implications these data could have.

It should be pointed out that, while the article reported unexpected and surprising results, it was not breaking any conceptual dogma. The idea that information could be transmitted from food in a form of a large organic molecule that would traverse into the human organism has been an integral part of the prion hypothesis, which brought a concept of foodborne infectious particles made only of proteins (prions, reviewed, for example in Peggion et al., 2016)). The prion hypothesis, for which Stanley Prusiner received a Nobel Prize in 1997 , is nowadays a biology textbook knowledge. Furthermore, cross-kingdom regulation by small RNAs was discovered in RNA silencing field already in its early years - long dsRNA expressed in bacteria could induce repression of worm genes with complementary sequences when worms were fed with such bacteria (Timmons and Fire, 1998). Furthermore, it was already well known in 2012 that feeding on a plant carrying an RNAi-inducing transgene can induce RNAi in nematodes, insects, or fungi (Baum et al., 2007; Huang et al., 2006; Mao et al., 2007; Nowara et al., 2010). Thus, the article by Zhang et al. was not bringing a major shift in existing paradigms but was rather extending knowledge by reporting an example of a miRNA activity transferred from plants to mammals through feeding.

\section{The critical issues}

The main controversy of the field of mammalian nutritional miRNAs is that its claims are inconsistent with the molecular mechanism of the mammalian miRNA pathway. Namely, there are three areas, which need to be further investigated and receive solid experimental support:

(i) Mechanism of the transport. It is unknown how plant miRNAs would be transported from the intestinal lumen into liver cells across cell membranes. Mammals have no systemic RNAi. miRNA pathway mobility is also limited. It is known that miRNAs can be released from cells and be detected in blood plasma (discussed above). While circulating miRNAs offer interesting biomarkers, their functional significance is still 
unclear and the mechanism of their sorting and release is also not understood. What is missing is the molecular mechanism transporting miRNAs from the intestinal lumen into the blood and into target cells.

(ii) Effector complex structure. It is unknown how plant miRNAs could regulate mammalian genes in mammalian cells. miRNA-mediated repression requires miRNAs to be loaded on AGO proteins. Thus, a plant miRNA operating in mammalian cells must either remain with its plant AGO protein, which would have to be compatible with mammalian components required for miRNA function, or be unloaded and reloaded onto a mammalian Argonaute protein. Importantly, plant miRNAs are methylated at their 3' end while mammalian miRNAs are not. Data by Zhang et al. suggest that plant miRNAs detected in mammalian serum are indeed methylated (Figure 1 in (Zhang et al., 2012a)). However, mammalian 3' small RNA methylation concerns only piRNAs, a distinct class of small RNAs produced from sense and antisense RNAs. piRNAs are longer (24-30 nt) than siRNAs or miRNAs and are loaded onto a distinct subfamily of Argonaute proteins named PIWI (reviewed in Peters and Meister, 2007). PIWI proteins are expressed in the germline but not in mammalian somatic cells. In contrast, miRNA and siRNA-binding Argonaute proteins (AGO subfamily) do not bind small RNAs methylated at the 3' end. (Tian et al., 2011). Yet, it has been reported that MIR168 is bound by murine AGO2.

(iii) Targeting stoichiometry. The canonical miRNA-mediated post-transcriptional regulation requires appropriate stoichiometry between miRNAs and their targets. However, the evidence that plant miRNAs would reach such physiologically active levels in liver cells is questionable. Plant miRNAs were detected in the serum at femtomolar concentrations $(\sim 3-16 \mathrm{fM})$, which was one order of magnitude less than the serum level of endogenous miRNAs (Zhang et al., 2012a). Total circulating miRNA concentration in human plasma ranges were estimated to be $68-250 \mathrm{fM}$ (Williams et al., 2013). At this concentration, human circulating miRNAs are considered unlikely to regulate gene expression by canonical miRNA activity as miRNAs are thought to require intracellular levels greater than 1000 copies per cell to exert measurable activity (Brown et al., 2007; Hafner et al., 2011). In fact, miR-122, a highly abundant miRNA regulating gene expression in the liver, has been estimated to be expressed at 120,000 molecules/ hepatocyte (one of the highest miRNA amounts/cell) while less expressed miR-33 and miR-16 were estimated to be expressed at 1,200 and 11,000 copies/hepatocyte, respectively (Bissels et al., 2009; Denzler et al., 2014). These numbers contrasts with estimates for xenogenic miRNAs. As 10 femtomoles of miRNAs are approximately $6 \times 10^{9}$ molecules, an extracellular concentration of $10 \mathrm{fM}$ corresponds to 6 molecules per nanoliter of serum. A somatic cell volume is up to several picoliters. Thus, unless there is a yet unknown active transport of circulating miRNAs into hepatocytes, xenogenic miRNAs entering the circulatory system have no capacity to regulate gene expression by canonical miRNA activity in the liver. However, Zhang et al. argue that MIR168a abundance in the liver tissue is equivalent to 853 copies per cell. The ongoing debate on stoichiometry, which is discussed later, has been inconclusive. Furthermore, a critical review of the results is compromised by the fact that some of the critical NGS datasets were not publicly released; hence, their analysis cannot be independently validated. 


\section{Exogenic miRNA transfer literature}

As of December 2016 when the EFSA report was being written (Paces et al., 2017), there were 227 citations of the original article, which I were reviewed. Among the 227, 51 original research articles were identified, which were related to xenogenic and circulating miRNAs. From these, I focus here on those directly relevant to the concept of plant-mammal miRNA transmission. Literature concerning the existence and function of xenogenic miRNAs includes:

- The original study (Zhang et al., 2012a). Using NGS, they identified plant miRNAs in mammalian sera. Unfortunately, the information provided was only partial. The entire data normalization was described as: For normalization, the sequencing frequency of each plant miRNA was normalized to the total amount of mammalian miRNAs. (Zhang et al., 2012a) while the availability of original sequencing data in a public repository was not indicated, thus not allowing for an independent assessment of sequence analysis and quantification. Different quantification strategy could be a potential source of discordance with other published data, which emerged in the literature (Chen et al., 2013).

- The patent: WO 2012135820A2 Edible transgenic plants as oral delivery vehicles for RNA-based therapeutics filled by Eric Lam, Rutgers University. The claim is based on an experiment where feeding rabbits or mice with transgenic tomato producing siRNA against $\mathrm{HCV}$ yielded detectable $\mathrm{HCV}$ siRNAs and tomato RNAs in mammalian blood and tissues. This work was not published in a peer review journal, the patent documentation contains a small RNA northern blot with a faint signal, RT-PCR detection of tomato RNAs in mammalian samples and analysis of RNAi-mediated repression in cultured HepG2 cells.

- A survey of human plasma for microRNA biomarkers in NGS datasets from human plasma revealed circulating RNAs appearing to originate from exogenous species including bacteria, fungi, and other species (Wang et al., 2012). Interestingly, authors could detect RNA fragments from common food where American samples exhibited higher abundance of corn over rice sequences while a control Chinese sample (SRR332232) showed a reversed trend (Wang et al., 2012). Authors also report detection of minuscule amounts of plant miRNAs in human samples 6-16 reads of MIR168 per sample were observed at total sequencing depths of 18-28 million reads; public availability of raw data was not indicated. Notably, authors also indicate low fraction of mapable sequences - they observed less than $1.5 \%$ of processed reads mapping to human miRNAs (Wang et al., 2012). Thus, calculating miRNA RPM values (reads per million) from the mapped reads or miRNA reads could yield two orders of magnitude differences.

- The same group, which published the original xenomiR study, recently published another article proposing that a plant miRNA MIR2911 from honeysuckle (Lonicera japonica), a traditional Chinese medicine herb, can traverse into mouse lungs and inhibit Influenza A (Zhou et al., 2015). In this case, MIR2911 concentration was estimated to reach $1 \mathrm{pM}$ in plasma after feeding mice honeysuckle decoction, which is the range of endogenous circulating miRNAs. MIR2911 was detected in the mouse by NGS (acc. no. GSE55268), qPCR, and in situ hybridization. The estimated amount of MIR2911 in lung cell was 
300-400, suggesting that MIR2911 operates within the physiological range of the canonical miRNA pathway (Zhou et al., 2015).

- Vicki Vance's group reported that oral administration of 2'-O-methylated mammalian miRNAs, which have tumor suppressor properties (miR-34a, miR-143, and miR145) results in reduced intestinal tumor burden in mice (Mlotshwa et al., 2015). This work is also linked to two issues patents and one pending patent application, all entitled "Compositions and Methods for the Modulation of Gene Expression in Plants": US patent application no. 13/282,680, Canadian patent no. 2,276,233 and Australian patent no. AU 2003/254052 B2.

- A third report of xenogenic miRNA regulation suggests that plant miRNA MIR159 is present in human plasma and its level inversely correlates with breast cancer incidence. MIR159 was identified in the serum by NGS (GSE55268). A synthetic mimic of MIR159 was capable of inhibiting cell proliferation in breast cancer cells but not non-cancerous mammary epithelial cells (Chin et al., 2016). Authors claim, "These results demonstrate for the first time that a plant miRNA can inhibit cancer growth in mammals."

- Work by Zhang et al. used miRNA target prediction in Arabidopsis and humans to assemble a hypothetical cross-species regulatory network, which they further analyzed. They proposed that the cross-species regulatory network contains three core module functionally linked to ion transport, metabolic process and stress response (Zhang et al., 2016). However, this study provides a strategy to explore possible cross-species miRNA regulations rather than providing evidence that such regulations exist.

Against these publications stands a number of reports, which question the idea of functional xenogenic miRNAs. For example:

- Analysis of 83 NGS datasets (identified in the report) revealed presence of plant-derived MIRNAs among which dominated MIR 168 from monocot plants. However, the highest observed ratio of plant miRNAs/animal miRNAs is $0.456 \%$, which is 10 times lower than a figure of $\sim 5 \%$ reported by Zhang et al. (Zhang et al., 2012a). These data suggests that the observed plant miRNAs in animal small RNA datasets can originate in the process of sequencing, and that accumulation of plant miRNAs via dietary exposure is not universal in animals (Zhang et al., 2012b).

- Snow et al. analyzed miRNA content in diets of humans, mice, and honeybees and examined transfer of several different miRNAs into the recipient organism: conserved and highly-expressed plant miRNAs (MIR156a, MIR159a and MIR169a) and conserved, abundant, and ubiquitous animal miRNA miR-21. Healthy human subjects routinely eating fruits replete in MIR156a, MIR159a and MIR169a had undetectable levels of plant-derived miRNAs in their plasma (as measured by qPCR). Similarly, miR-21 levels were negligible in plasma or tissues of miR-21/- mice after oral diets containing miR-21. The same was observed for MIR156a, MIR159a and MIR169a in mice and honeybee tissues. This study concludes that: " horizontal delivery of microRNAs via typical dietary ingestion is neither a robust nor a frequent mechanism to maintain steady-state microRNA levels in a variety of model animal organisms, thus defining the biological limits of these molecules in vivo." (Snow et al., 2013)

- Importantly, an attempt to replicate the claims in the original xenogenic miRNA paper failed (Dickinson et al., 2013). This article prompted a comment by authors of the original 


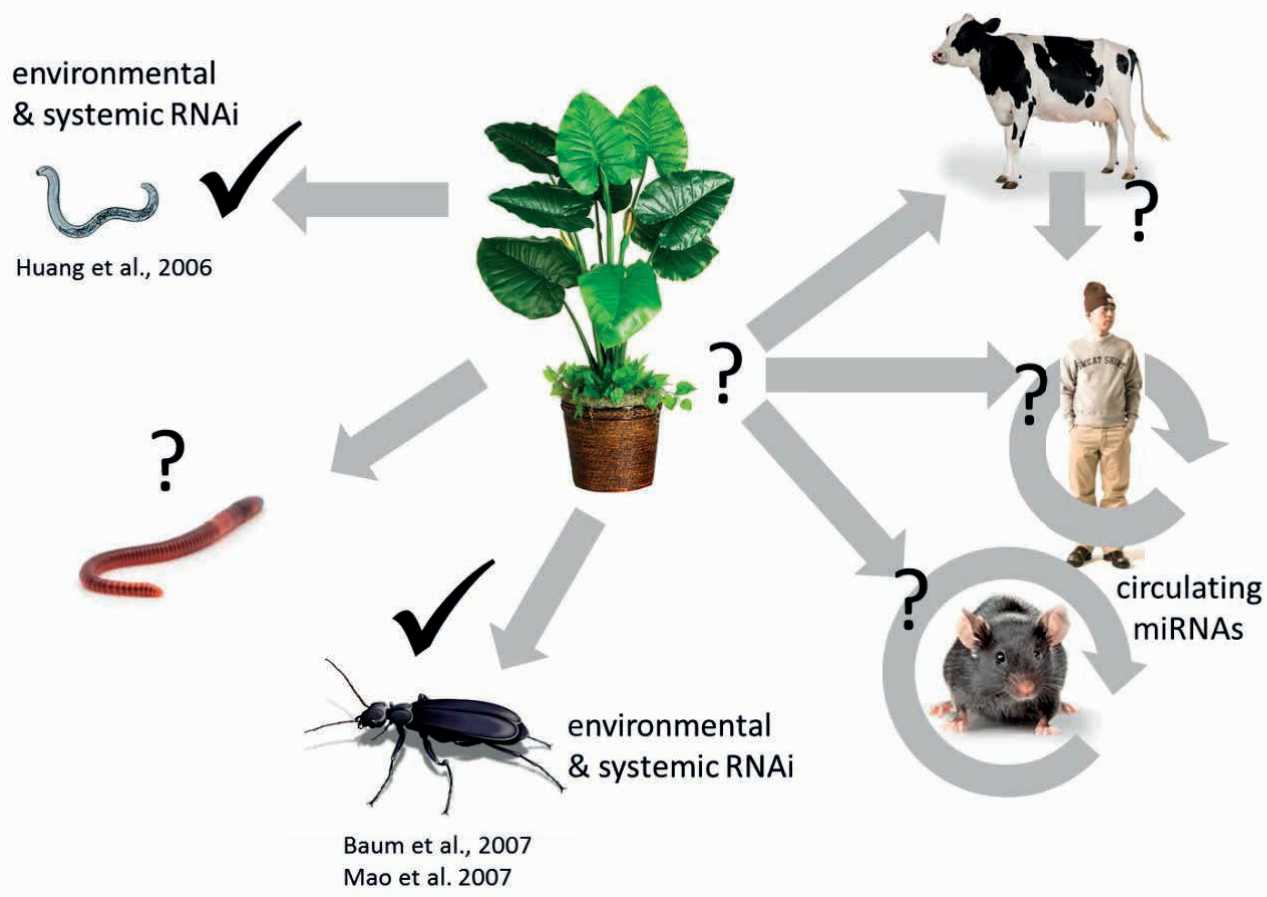

Figure 4 Mobile RNA silencing

The scheme depicts confirmed trans-species/trans-kingdom silencing where silencing of parasitic nematodes or insect pests can be induced by RNA produced in plants. Transmission of miRNAs and induction of silencing into other species remains controversial although existence of circulating miRNAs in humans and mice is well documented.

xenogenic paper pointing out several discrepancies in data and that experimental design used by Dickinson et al. for NGS analysis was suboptimal and could produce artifacts (Chen et al., 2013). Unfortunately, one cannot reanalyze both datasets because only the one from Dickinson et al. was made available (SRP028401).

- A feeding study on Macaca nemestrina failed to provide support for xenogenic miRNAs in blood plasma in response to dietary intake of a plant miRNA-rich food source. Putative xenogenic miRNAs were detected real-time quantitative PCR and droplet digital PCR (Witwer et al., 2013). Detected levels of miRNAs were minimal and/or amplification was non-specific. Detection was largely unreliable for plant miRNAs 156, 160, $166,167,168$ and 172 despite the relative abundance of all but miR172 in a soy- and fruit-substance that was administered to the macaque subjects (Witwer et al., 2013).

- Bioinformatic analysis of NGS data for diet-derived miRNAs in deep-sequencing libraries also did not provide a support for the "xenomiR hypothesis". Authors provide evidence that cross-contamination during library preparation can be a source of exogenous RNAs and that such a cross-contamination could be identified in an NGS study of Amphioxus performed by the group, which published the first xenogenic miRNA article 
in 2012 (Tosar et al., 2014). The original data from the Zhang et al. 2012 article could not be examined for cross-contamination. Tossar et al. conclude that cross-contamination between samples from the same organism can go completely unnoticed, possibly affecting conclusions derived from NGS transcriptomics (Tosar et al., 2014).

Taken together, the field remains split since the original report (Zhang et al., 2012a). The essential questions concerning the existence of the proposed mechanism emerged already in 2012. Further research is necessary to clarify the basis of the aforementioned contradictory observations. At the moment, there is no consensus regarding specific aspects of the xenomiR hypothesis, i.e.:

- the mechanism of transport of a plant miRNAs from food into the blood.

- amounts of plant miRNAs reaching the mammalian circulation system and tissues

- the nature of the ribonucleoprotein complexes, in which they exist in the plasma

- the mechanism of entrance (accumulation?) of xenomiRs into cells

- the nature of the ribonucleoprotein complexes, in which xenomiRs exist in the cell Remarkably, the simplicity of the xenomiR hypothesis where an oral uptake of miRNAs in food results in post-transcriptional repression in tissues contrasts with more than a decade of development of small RNA-based therapeutics, which still struggles with efficiency of uptake, distribution, and targeting. However, the current stage of (incomplete) knowledge precludes strong conclusions regarding the future of the xenomiR hypothesis.

\section{Conclusions}

Mobile RNA silencing exists in plants and some animals (Fig. 4). RNA silencing, and RNAi in particular, operates with unique (though not absolute) selectivity and is being explored in many different ways in biomedicine and biotechnology. In some cases, RNA silencing can spread across cellular boundaries, which can extend into systemic silencing. Systemic silencing is restricted to specific taxons and RNA silencing pathways. While the framework for understanding the molecular basis of systemic silencing in different model systems was established, the knowledge is still fragmented and far from complete. Even less clear is the horizontal transmission of RNA silencing, especially into species, where primarily cell-autonomous silencing is expected.

\section{Acknowledgement}

I would like to thank my colleagues Jan Paces, Miloslav Nic, Tomas Novotny, and Eliska Sovobodova for help with collecting literature for the review. The review content was produced under a contract OC/EFSA/GMO/2015/01-CT 01 with European Food Safety Authority (EFSA); the opinions expressed are those of the contractor only and do not represent EFSA's official position. Publication of the review was funded by LO1220 and LM2015063 by the Ministry of Education, Youth and Sports. 


\section{References}

Alsaweed, M., Lai, C.T., Hartmann, P.E., Geddes, D.T., and Kakulas, F. (2016a). Human Milk Cells and Lipids Conserve Numerous Known and Novel miRNAs, Some of Which Are Differentially Expressed during Lactation. Plos One 11.

Alsaweed, M., Lai, C.T., Hartmann, P.E., Geddes, D.T., and Kakulas, F. (2016b). Human Milk Cells Contain Numerous miRNAs that May Change with Milk Removal and Regulate Multiple Physiological Processes. International Journal of Molecular Sciences 17.

Alsaweed, M., Lai, C.T., Hartmann, P.E., Geddes, D.T., and Kakulas, F. (2016c). Human milk miRNAs primarily originate from the mammary gland resulting in unique miRNA profiles of fractionated milk. Scientific Reports 6 .

Arroyo, J.D., Chevillet, J.R., Kroh, E.M., Ruf, I.K., Pritchard, C.C., Gibson, D.F., Mitchell, P.S., Bennett, C.F., Pogosova-Agadjanyan, E.L., Stirewalt, D.L., et al. (2011). Argonaute2 complexes carry a population of circulating microRNAs independent of vesicles in human plasma. Proc Natl Acad Sci U S A 108, 5003-5008.

Baglio, S.R., Rooijers, K., Koppers-Lalic, D., Verweij, F.J., Pérez Lanzón, M., Zini, N., Naaijkens, B., Perut, F., Niessen, H.W.M., Baldini, N., et al. (2015). Human bone marrow- and adipose-mesenchymal stem cells secrete exosomes enriched in distinctive miRNA and tRNA species. Stem Cell Research and Therapy 6.

Baier, S.R., Nguyen, C., Xie, F., Wood, J.R., and Zempleni, J. (2014). MicroRNAs Are Absorbed in Biologically Meaningful Amounts from Nutritionally Relevant Doses of Cow Milk and Affect Gene Expression in Peripheral Blood Mononuclear Cells, HEK-293 Kidney Cell Cultures, and Mouse Livers. Journal of Nutrition 144, 1495-1500.

Baum, J.A., Bogaert, T., Clinton, W., Heck, G.R., Feldmann, P., Ilagan, O., Johnson, S., Plaetinck, G., Munyikwa, T., Pleau, M., et al. (2007). Control of coleopteran insect pests through RNA interference. Nat Biotechnol 25, 1322-1326.

Benmoussa, A., Lee, C.H.C., Laffont, B., Savard, P., Laugier, J., Boilard, E., Gilbert, C., Fliss, I., and Provost, P. (2016). Commercial Dairy Cow Milk microRNAs Resist Digestion under Simulated Gastrointestinal Tract Conditions. Journal of Nutrition 146, 2206-2215.

Bhatia, V., Bhattacharya, R., Uniyal, P.L., Singh, R., and Niranjan, R.S. (2012). Host Generated siRNAs Attenuate Expression of Serine Protease Gene in Myzus persicae. Plos One 7, e46343-e46343.

Bissels, U., Wild, S., Tomiuk, S., Holste, A., Hafner, M., Tuschl, T., and Bosio, A. (2009). Absolute quantification of microRNAs by using a universal reference. RNA 15, 2375-2384.

Brown, B.D., Gentner, B., Cantore, A., Colleoni, S., Amendola, M., Zingale, A., Baccarini, A., Lazzari, G., Galli, C., and Naldini, L. (2007). Endogenous microRNA can be broadly exploited to regulate transgene expression according to tissue, lineage and differentiation state. Nat Biotechnol 25, 1457-1467.

Buck, A.H., Coakley, G., Simbari, F., McSorley, H.J., Quintana, J.F., Le Bihan, T., Kumar, S., Abreu-Goodger, C., Lear, M., Harcus, Y., et al. (2014). Exosomes secreted by nematode parasites transfer small RNAs to mammalian cells and modulate innate immunity. Nature Communications 5 .

Chen, X., Zen, K., and Zhang, C.-Y. (2013). Lack of detectable oral bioavailability of plant microRNAs after feeding in mice Reply. Nature Biotechnology 31, 967-969. 
Chera, S., de Rosa, R., Miljkovic-Licina, M., Dobretz, K., Ghila, L., Kaloulis, K., and Galliot, B. (2006). Silencing of the hydra serine protease inhibitor Kazall gene mimics the human SPINK1 pancreatic phenotype. J Cell Sci 119, 846-857.

Chevillet, J.R., Kang, Q., Ruf, I.K., Briggs, H.A., Vojtech, L.N., Hughes, S.M., Cheng, H.H., Arroyo, J.D., Meredith, E.K., Gallichotte, E.N., et al. (2014). Quantitative and stoichiometric analysis of the microRNA content of exosomes. Proc Natl Acad Sci U S A 111, 14888-14893.

Chim, S.S., Shing, T.K., Hung, E.C., Leung, T.Y., Lau, T.K., Chiu, R.W., and Lo, Y.M. (2008). Detection and characterization of placental microRNAs in maternal plasma. Clinical chemistry 54 , 482-490.

Chin, A.R., Fong, M.Y., Somlo, G., Wu, J., Swiderski, P., Wu, X., and Wang, S.E. (2016). Cross-kingdom inhibition of breast cancer growth by plant miR159. Cell Research 26, 217-228.

Denzler, R., Agarwal, V., Stefano, J., Bartel, D.P., and Stoffel, M. (2014). Assessing the ceRNA hypothesis with quantitative measurements of miRNA and target abundance. Mol Cell 54, 766-776.

Dickinson, B., Zhang, Y., Petrick, J.S., Heck, G., Ivashuta, S., and Marshall, W.S. (2013). Lack of detectable oral bioavailability of plant microRNAs after feeding in mice. Nature Biotechnology 31, 965-967.

Dismuke, W.M., Challa, P., Navarro, I., Stamer, W.D., and Liu, Y.T. (2015). Human aqueous humor exosomes. Experimental Eye Research 132, 73-77.

Geldhof, P., Visser, A., Clark, D., Saunders, G., Britton, C., Gilleard, J., Berriman, M., and Knox, D. (2007). RNA interference in parasitic helminths: current situation, potential pitfalls and future prospects. Parasitology 134, 609-619.

Hafner, M., Renwick, N., Brown, M., Mihailovic, A., Holoch, D., Lin, C., Pena, J.T., Nusbaum, J.D., Morozov, P., Ludwig, J., et al. (2011). RNA-ligase-dependent biases in miRNA representation in deep-sequenced small RNA cDNA libraries. RNA 17, 1697-1712.

Himber, C., Dunoyer, P., Moissiard, G., Ritzenthaler, C., and Voinnet, O. (2003). Transitivity-dependent and -independent cell-to-cell movement of RNA silencing. The EMBO journal 22, 4523-4533.

Huang, G., Allen, R., Davis, E.L., Baum, T.J., and Hussey, R.S. (2006). Engineering broad root-knot resistance in transgenic plants by RNAi silencing of a conserved and essential root-knot nematode parasitism gene. Proc Natl Acad Sci U S A 103, 14302-14306.

Huang, X.Y., Yuan, T.Z., Tschannen, M., Sun, Z.F., Jacob, H., Du, M.J., Liang, M.H., Dittmar, R.L., Liu, Y., Liang, M.Y., et al. (2013). Characterization of human plasma-derived exosomal RNAs by deep sequencing. BMC Genomics 14, 319-319.

Izumi, H., Kosaka, N., Shimizu, T., Sekine, K., Ochiya, T., and Takase, M. (2012). Bovine milk contains microRNA and messenger RNA that are stable under degradative conditions. Journal of Dairy Science 95, 4831-4841.

Izumi, H., Tsuda, M., Sato, Y., Kosaka, N., Ochiya, T., Iwamoto, H., Namba, K., and Takeda, Y. (2015). Bovine milk exosomes contain microRNA and mRNA and are taken up by human macrophages. Journal of Dairy Science 98, 2920-2933.

Kola, V.S.R., Renuka, P., Padmakumari, A.P., Mangrauthia, S.K., Balachandran, S.M., Babu, V.R., and Madhav, M.S. (2016). Silencing of CYP6 and APN Genes Affects the Growth and Development of Rice Yellow Stem Borer, Scirpophaga incertulas. Frontiers in Physiology 7, 20-20.

Kropp, J., Salih, S.M., and Khatib, H. (2014). Expression of microRNAs in bovine and human pre-implantation embryo culture media. Frontiers in Genetics 5, 91-91. 
Kumar, L., Verma, S., Vaidya, B., and Gupta, V. (2015). Exosomes: Natural Carriers for siRNA Delivery. Current Pharmaceutical Design 21, 4556-4565.

Kuruppath, S., Kumar, A., Modepalli, V.N., Ngo Khanh, P., Gras, S.L., and Lefevre, C. (2013). Buffalo Milk Transcriptomics. Buffalo Bulletin 32, 796-804.

Lasser, C. (2012). Exosomal RNA as biomarkers and the therapeutic potential of exosome vectors. Expert Opinion on Biological Therapy 12, S189-S197.

Lawrie, C.H., Gal, S., Dunlop, H.M., Pushkaran, B., Liggins, A.P., Pulford, K., Banham, A.H., Pezzella, F., Boultwood, J., Wainscoat, J.S., et al. (2008). Detection of elevated levels of tumour-associated microRNAs in serum of patients with diffuse large B-cell lymphoma. British journal of haematology 141, 672-675.

Mansoor, S., Amin, I., Hussain, M., Zafar, Y., and Briddon, R.W. (2006). Engineering novel traits in plants through RNA interference. Trends Plant Sci 11, 559-565.

Mao, Y.B., Cai, W.J., Wang, J.W., Hong, G.J., Tao, X.Y., Wang, L.J., Huang, Y.P., and Chen, X.Y. (2007). Silencing a cotton bollworm P450 monooxygenase gene by plant-mediated RNAi impairs larval tolerance of gossypol. Nat Biotechnol 25, 1307-1313.

Melnyk, C.W., Molnar, A., and Baulcombe, D.C. (2011). Intercellular and systemic movement of RNA silencing signals. The EMBO journal 30, 3553-3563.

Mlotshwa, S., Pruss, G.J., MacArthur, J.L., Endres, M.W., Davis, C., Hofseth, L.J., Pena, M.M., and Vance, V. (2015). A novel chemopreventive strategy based on therapeutic microRNAs produced in plants. Cell Research 25, 521-524.

Newmark, P.A., Reddien, P.W., Cebria, F., and Sanchez Alvarado, A. (2003). Ingestion of bacterially expressed double-stranded RNA inhibits gene expression in planarians. Proc Natl Acad Sci U S A 100 Suppl 1, 11861-11865.

Nowara, D., Gay, A., Lacomme, C., Shaw, J., Ridout, C., Douchkov, D., Hensel, G., Kumlehn, J., and Schweizer, P. (2010). HIGS: host-induced gene silencing in the obligate biotrophic fungal pathogen Blumeria graminis. Plant Cell 22, 3130-3141.

Orii, H., Mochii, M., and Watanabe, K. (2003). A simple "soaking method" for RNA interference in the planarian Dugesia japonica. Dev Genes Evol 213, 138-141.

Paces, J., Nic, M., Novotny, T., and Svoboda, P. (2017). Literature review of baseline information to support the risk assessment of RNAi-based GM plants. EFSA Supporting Publications 14, 315 .

Peggion, C., Bertoli, A., and Sorgato, M.C. (2016). Almost a century of prion protein(s): From pathology to physiology, and back to pathology. Biochem Biophys Res Commun.

Pegtel, D.M., van de Garde, M.D.B., and Middeldorp, J.M. (2011). Viral miRNAs exploiting the endosomal-exosomal pathway for intercellular cross-talk and immune evasion. Biochimica Et Biophysica Acta-Gene Regulatory Mechanisms 1809, 715-721.

Peters, L., and Meister, G. (2007). Argonaute proteins: Mediators of RNA silencing. Molecular Cell 26, 611-623.

Rak, J. (2013). Extracellular vesicles - biomarkers and effectors of the cellular interactome in cancer. Frontiers in Pharmacology 4, 21-21.

Snow, J.W., Hale, A.E., Isaacs, S.K., Baggish, A.L., and Chan, S.Y. (2013). Ineffective delivery of diet-derived microRNAs to recipient animal organisms. Rna Biology 10, 1107-1116.

Sun, Q., Chen, X., Yu, J., Zen, K., Zhang, C.-Y., and Li, L. (2013). Immune modulatory function of abundant immune-related microRNAs in microvesicles from bovine colostrum. Protein \& Cell 4, $197-210$. 
Tabara, H., Grishok, A., and Mello, C.C. (1998). RNAi in C. elegans: soaking in the genome sequence. Science 282, 430-431.

Tian, Y., Simanshu, D.K., Ma, J.B., and Patel, D.J. (2011). Structural basis for piRNA 2'-O-methylated 3'-end recognition by Piwi PAZ (Piwi/Argonaute/Zwille) domains. Proc Natl Acad Sci U S A 108, 903-910.

Timmons, L., and Fire, A. (1998). Specific interference by ingested dsRNA. Nature 395, 854.

Tomoyasu, Y., Miller, S.C., Tomita, S., Schoppmeier, M., Grossmann, D., and Bucher, G. (2008). Exploring systemic RNA interference in insects: a genome-wide survey for RNAi genes in Tribolium. Genome Biol 9, R10.

Tosar, J.P., Rovira, C., Naya, H., and Cayota, A. (2014). Mining of public sequencing databases supports a non-dietary origin for putative foreign miRNAs: underestimated effects of contamination in NGS. Rna-a Publication of the Rna Society 20, 754-757.

Turchinovich, A., Tonevitsky, A.G., and Burwinkel, B. (2016). Extracellular miRNA: A Collision of Two Paradigms. Trends Biochem Sci 41, 883-892.

Turchinovich, A., Weiz, L., Langheinz, A., and Burwinkel, B. (2011). Characterization of extracellular circulating microRNA. Nucleic Acids Research 39, 7223-7233.

Wang, K., Li, H., Yuan, Y., Etheridge, A., Zhou, Y., Huang, D., Wilmes, P., and Galas, D. (2012). The Complex Exogenous RNA Spectra in Human Plasma: An Interface with Human Gut Biota? Plos One 7.

Wee, L.M., Flores-Jasso, C.F., Salomon, W.E., and Zamore, P.D. (2012). Argonaute Divides Its RNA Guide into Domains with Distinct Functions and RNA-Binding Properties. Cell 151, 1055-1067. Whangbo, J.S., and Hunter, C.P. (2008). Environmental RNA interference. Trends Genet 24, 297-305. Williams, Z., Ben-Dov, I.Z., Elias, R., Mihailovic, A., Brown, M., Rosenwaks, Z., and Tuschl, T. (2013). Comprehensive profiling of circulating microRNA via small RNA sequencing of cDNA libraries reveals biomarker potential and limitations. Proc Natl Acad Sci U S A 110, 4255-4260.

Witwer, K.W., McAlexander, M.A., Queen, S.E., and Adams, R.J. (2013). Real-time quantitative PCR and droplet digital PCR for plant miRNAs in mammalian blood provide little evidence for general uptake of dietary miRNAs Limited evidence for general uptake of dietary plant xenomiRs. Rna Biology 10, 1080-1086.

Wu, G.P., Yang, G.H., Zhang, R.X., Xu, G.Y., Zhang, L., Wen, W., Lu, J.B., Liu, J.Y., and Yu, Y. (2015). Altered microRNA Expression Profiles of Extracellular Vesicles in Nasal Mucus From Patients With Allergic Rhinitis. Allergy Asthma \& Immunology Research 7, 449-457.

Xi, Y., Jiang, X., Li, R., Chen, M., Song, W., and Li, X. (2016). The levels of human milk microRNAs and their association with maternal weight characteristics. European Journal of Clinical Nutrition 70, 445-449.

Xu, W., and Han, Z. (2008). Cloning and phylogenetic analysis of sid-1-like genes from aphids. J Insect Sci 8, 1-6.

Yoon, Y.J., Kim, O.Y., and Gho, Y.S. (2014). Extracellular vesicles as emerging intercellular communicasomes. BMB Reports 47, 531-539.

Zhang, H., Li, Y., Liu, Y., Liu, H., Wang, H., Jin, W., Zhang, Y., Zhang, C., and Xu, D. (2016). Role of plant MicroRNA in cross-species regulatory networks of humans. Bmc Systems Biology 10.

Zhang, L., Hou, D.X., Chen, X., Li, D.H., Zhu, L.Y., Zhang, Y.J., Li, J., Bian, Z., Liang, X.Y., Cai, X., et al. (2012a). Exogenous plant MIR168a specifically targets mammalian LDLRAP1: evidence of cross-kingdom regulation by microRNA. Cell Research 22, 107-126. 
Zhang, Y., Wiggins, B.E., Lawrence, C., Petrick, J., Ivashuta, S., and Heck, G. (2012b). Analysis of plant-derived miRNAs in animal small RNA datasets. Bmc Genomics 13.

Zhou, Z., Li, X., Liu, J., Dong, L., Chen, Q., Liu, J., Kong, H., Zhang, Q., Qi, X., Hou, D., et al. (2015). Honeysuckle-encoded atypical microRNA2911 directly targets influenza A viruses. Cell Research 25, 39-49. 